The World Health Organization has expanded the microscopic forms of urothelial carcinomas to include several unusual histological variants. The importance of the recognition of these unusual forms is related to: a) different prognosis; b) different therapeutic approach; and, c) the possibility of misinterpretation by the pathologist.

An important example is the small cell carcinoma with neuroendocrine differentiation. The importance of an accurate diagnosis of this variant of urothelial carcinoma is its response to newer chemotherapy protocols. The histological variants of urothelial carcinoma includes: nested variant, urothelial carcinoma with small tubules, microcystic urothelial carcinoma, micropapillary variant, lymphepithelioma-like, sarcomatoid carcinoma (carcinosarcoma), small cell carcinoma, plasmacytoid carcinoma, urothelial carcinoma with rhabdoid features, urothelial carcinoma with clear cytoplasm (glycogen-rich), urothelial carcinoma with trophoblastic differentiation, urothelial carcinoma with unusual stromal reactions, osteoclast-rich undifferentiated carcinoma, and giant cell carcinoma (1).

The study by Keck et al. based on a large series of plasmacytoid carcinoma showed that this tumor is an aggressive variant of bladder cancer with molecular features of advanced bladder cancer and evidence of WNT pathway activation in some of the cases.

\title{
References
}

1. Epstein JI, Reuter VE, Amin MB: Biopsy Interpretation of the Bladder, 2nd ed. Philadelphia, Lippincott Williams \& Wilkins. 2010.

\author{
Dr. Athanase Billis \\ Full-Professor of Pathology \\ State University of Campinas, Unicamp \\ Campinas, São Paulo, Brazil \\ E-mail: athanase@fcm.unicamp.br
}

\section{RECONSTRUCTIVE UROLOGY}

doi: 10.1590/S1677-55382010000600024

\section{Volar onlay urethroplasty for reconstruction of female urethra in recurrent stricture disease Gozzi C, Roosen A, Bastian PJ, Karl A, Stief C, Tritschler S \\ Department of Urology, Klinikum Großhadern, Ludwig Maximilians University of Munich, Munich, Germany}

BJU Int. 2010 Nov 17 [Epub ahead of print]

Objective: To report our experience with a new and simple method of urethral repair with a volar onlay of free labium minus graft. Strictures of the female urethra are rare, and it is well accepted that the therapeutic options of dilation and urethrotomy are not lasting solutions as a result of their high recurrence rates. However, there is no consensus regarding the best way to reconstruct the female urethra in the case of stricture disease.

Patients and Methods: Four consecutive female patients with a long lasting history of recurrent urethral strictures underwent open urethroplasty with a volar situated free split thickness epidermal graft from the labium minus. The surgical technique is described and a short-term follow-up is presented. 
Results: Operating time was 40-140 min (mean $105 \mathrm{~min}$ ), and the graft measured between $2 \times 1.5 \mathrm{~cm}$ and 3 $\times 2.5 \mathrm{~cm}$. Follow-up time was 11-19 months. Maximum urinary flow rate could be improved from a baseline of $9.4-11.2 \mathrm{~mL} / \mathrm{s}$ (preoperatively, after intermittent use of dilation) to $19-23 \mathrm{~mL} / \mathrm{s}$. Postvoid residual urine volume was $0-50 \mathrm{~mL}$ preoperatively and no postvoid residual urine volume postoperatively. Urinary catheters were removed after 21 days. Urinary stress incontinence did not occur postoperatively. There were found no complications related to the graft donor site.

Conclusions: The reported data concerning a new therapeutic approach for the treatment of recurrent female urethral stricture show that a volar onlay urethroplasty represents a feasible, safe and simple surgical method. Larger series with long-term follow-up are needed for further evaluation.

doi: $10.1590 / S 1677-55382010000600025$

\section{Vaginal flap urethroplasty for wide female stricture disease}

Simonato A, Varca V, Esposito M, Carmignani G

Clinica Urologica L. Giuliani, Ospedale San Martino, Università degli Studi di Genova, Genova, Italy

J Urol. 2010; 184: 1381-5

Purpose: As in men, female urethral stricture disease is often treated with repeat urethral dilation or internal urethrotomy but not always with good results. In nonresponsive cases surgical treatment may be useful but only a few cases are reported in the literature. We present our single institution experience with urethral reconstruction in 6 patients using an alternative vaginal inlay flap technique inspired by the Orandi technique.

Materials and Methods: We treated 6 women with urethral stricture. In 5 patients stricture involved the entire middle and distal urethra, and in 1 it also involved the proximal urethra with bilateral hydronephrosis. Patients underwent urethral reconstruction using a vaginal flap with a lateral vascular pedicle that maintains the vascular axis. The flap was partially de-epithelialized to favor tissue cicatrix formation where the sutures are placed and avoid fistula formation.

Results: Mean followup was 70.8 months. Normal micturition was achieved after catheter removal in all patients. Post-void residual urine was measured postoperatively in 3 patients. One patient had significant post-void residual urine and required intermittent self-catheterization. The remaining 5 patients required no additional treatment.

Conclusions: Using the vaginal wall to reconstruct large segments of the female urethra is simple and appears to have good results. Our technique preserves the vascular axis of the flap and protects the sutures. More contributions to the existing literature are needed before any further conclusions can be drawn.

\section{Editorial Comment}

Repair of female urethral stricture disease is difficult. First, the disease is less common than male urethral stricture disease making the surgeon less familiar with the technique. Second, the shorter urethra and proximity to the vaginal mucosa allows for little margin of error. Perhaps the multitude of surgical approaches described attests to the quest to find a universally acceptable approach. Dividing the urethra along its volar aspect (the vaginal rather than clitoral body side) is preferable for many reasons. First, it avoids the majority of the sphincter fibers. These fibers follow an omega shape and are more prominent on the clitoral side. Second, a volar dissection is familiar to most urologists as the dissection for most anti-incontinence procedures is done in this area. Third, it avoids dissection of the urethra off the clitoral bodies - a dissection unfamiliar to urologists.

These two articles present descriptions of modifications of the volar urethroplasty in women. In Gozzi et al, the authors describe a suburethral incision followed by dissection of the vaginal flap off the urethra, a 
volar urethrotomy and excision of all scarred tissue. A labia minora graft is then harvested, thinned, and grafted ventrally, using the periurethral tissue as a graft bed. The vaginal flap is closed. In contrast, the Simonato et al group describes an approach that borrows heavily from the Orandi urethroplasty well-known in reconstruction of penile urethral stricture disease. A laterally-based vaginal flap is created and the middle portion is de-epithelialized. This essentially creates a medially located island flap which is then rolled onto the ventral urethrotomy. The remaining (lateral) vaginal flap is closed over the urethra.

Both of these approaches are attractive in the fact that they use a volar approach and borrow from reconstructive principles used in male urethral stricture surgery. Both approaches are most appropriate in the distal to middle third of the urethra. The proximal third remains a higher risk area due to the deeper dissection and the prominence of bladder neck sphincter fibers. Small patient numbers and limited follow-up may limit the external validity of the results of these two series.

\author{
Dr. Sean P. Elliott \\ Department of Urology Surgery \\ University of Minnesota \\ Minneapolis, Minnesota, USA \\ E-mail:selliott@umn.edu
}

\title{
UROLOGICAL ONCOLOGY
}

doi: $10.1590 / S 1677-55382010000600026$

Clinical outcome in a contemporary series of restaged patients with clinical T1 bladder cancer Dalbagni G, Vora K, Kaag M, Cronin A, Bochner B, Donat SM, Herr HW

Division of Urology, Memorial Sloan-Kettering Cancer Center, New York, NY, USA

Eur. Urol. 2009; 56: 903-910

Objectives: To evaluate the indications for early and deferred cystectomy and to report the impact of this tailored approach on survival.

Design, Setting, and Participants: We retrospectively studied 523 patients seen at our institution who were initially diagnosed with T1 disease between 1990 and 2007.

Measurements: Variables analyzed included age, gender, multifocality, multifocal T1 disease, carcinoma in situ, grade, recurrence rate, and restaging status. End points were overall and disease-specific survival.

Results and Limitations: A restaging transurethral resection (TUR) was performed in 523 patients. Of the patients who underwent restaging, $106(20 \%)$ were upstaged to muscle-invasive disease and 417 patients were considered true clinical T1 (cT1); 84 of the latter group underwent immediate cystectomy. The median follow-up for survivors was $4.3 \mathrm{yr}$. The cumulative incidence of disease-specific death at $5 \mathrm{yr}$ was $8 \%$ (95\% confidence interval [CI], 5-13\%), 10\% (95\% CI, 5-17\%), and 44\% (95\% CI, 35-56\%) for those restaged with lower than $\mathrm{T} 1$, T1, and T2 disease, respectively. Immediate cystectomy was more likely in patients with cT1 disease at restaging than in those with disease lower than $\mathrm{cT} 1$, but there were no other obvious differences in clinical characteristics between those with and without immediate cystectomy. Survival was not statistically different for patients who underwent an immediate cystectomy versus those who were maintained on surveillance with deferred cystectomy if deemed appropriate. Of 333 patients who did not undergo immediate cystectomy, 59 had a deferred cystectomy, and the likelihood of deferred cystectomy was greater in those with T1 disease on restaging TUR (hazard ratio: 2.40; 95\% CI, 1.43-4.01; $\mathrm{p}=0.001$ ). 
Conclusions: Restaging TUR should be performed in patients diagnosed with cT1 bladder cancer to improve staging accuracy. Patients with T1 disease on restaging are at higher risk of progression and should be considered for early cystectomy.

\title{
Editorial Comment
}

The timing of cystectomy in T1 bladder cancer is a matter of debate since years. Here, the authors from a tertiary referral center present their series of 523 patients and analyze variables which may help with the decision to remain conservatively, or proceed with radical surgical therapy. Interestingly, re-TUR was performed in all patients and yielded a high rate of $20 \%$ upstaging to muscle-invasive disease. If true $\mathrm{T} 1$ was considered, the disease-specific mortality at 5 years was $10 \%$, with no survival differences between those patients undergoing early cystectomy versus those with no or deferred cystectomy. Clearly, these data support an initial conservative approach in select patient with true pT1. In any case, a re-TUR is mandatory.

\author{
Dr. Andreas Bohle \\ Professor of Urology \\ HELIOS Agnes Karll Hospital \\ Bad Schwartau, Germany \\ E-mail:boehle@urologie-bad-schwartau.de
}

doi: $10.1590 / S 1677-55382010000600027$

\section{Outcome predictors of radical prostatectomy in patients with prostate-specific antigen greater than $20 \mathrm{ng} / \mathrm{ml}$ : A European multi-institutional study of 712 patients}

Spahn M, Joniau S, Gontero P, Fieuws S, Marchioro G, Tombal B, Kneitz B, Hsu CY, Van Der Eeckt K, Bader P, Frohneberg D, Tizzani A, Van Poppel H

University Hospital Würzburg, Department of Urology and Pediatric Urology, Germany; Community Hospital Karlsruhe, Department of Urology, Karlsruhe, Germany

Eur. Urol. 58: 1-7, 2010

Background: Prostate cancer ( $\mathrm{PCa}$ ) patients with pretreatment prostate-specific antigen (PSA) $>20 \mathrm{ng} / \mathrm{ml}$ have a high risk of biochemical and clinical failure and even cancer-related death after local therapy. Pretreatment predictors of outcome after radical prostatectomy (RP) in this patient group are necessary.

Objective: Our aim was to assess how the use of additional high-risk factors (biopsy Gleason score $[\mathrm{bGS}]>/=8$ or clinical stage 3-4) can improve prediction of treatment failure and cancer-related death after RP in patients with PSA $>20$.

Design, Setting, and Participants: In a retrospective multicentre cohort study from six European centres between 1987 and 2005, 712 patients with PSA $>20 \mathrm{ng} / \mathrm{ml}$ underwent RP and bilateral pelvic lymphadenectomy.

Measurements: Subgroups were analysed to determine the relationship between the number of high-risk factors and histopathology, biochemical progression-free survival, clinical evidence of progressive disease, prostate cancer-specific mortality (PCSM), and overall mortality. Kaplan-Meier analysis with log-rank test and Cox multivariable analysis were applied.

Results and Limitations: Median follow-up was 77 mo. The number of high-risk factors was significantly associated with unfavourable histopathology. Among patients with only PSA $>20 \mathrm{ng} / \mathrm{ml}, 33 \%$ had pT2 PCa, 57.9\% had bGS $<7,54 \%$ had negative surgical margins, and $85 \%$ were lymph node negative (pN0), whereas among patients with all three high-risk factors, $4.5 \%$ had pT2 PCa, 2.3\% had bGS $<7,20.5 \%$ had negative margins, 
and $49 \%$ were $\mathrm{pN} 0(\mathrm{p}<0.001)$. The strongest predictor of progression and mortality was bGS. PSA $>20 \mathrm{ng} / \mathrm{ml}$ associated with bGS $</=7$ resulted in 10 -yr PCSM of $5 \%$; when associated with bGS $>/=8$, PCSM was $35 \%$. The main limitations of the study were retrospective design and varying treatment modalities.

Conclusions: PCa patients with PSA $>20 \mathrm{ng} / \mathrm{ml}$ have varying risk levels of disease progression and PCSM. Considering additional risk factors further stratifies this group into four subgroups that can guide the clinician in preoperative patient counselling.

\section{Editorial Comment}

Surgical therapy in patients with prostate cancer and a PSA $>20 \mathrm{ng} / \mathrm{ml}$ is a matter of debate. Most patients are considered high-risk and receive either hormonal therapy alone or are referred to external beam radiation therapy. The authors from this multi-institutional study analyze their 712 patients with PSA $>20 \mathrm{ng} / \mathrm{ml}$ who underwent radical prostatectomy. Of this group, roughly $40 \%$ had Gleason score $>7,50 \%$ had positive surgical margins and $15 \%$ were node-positive. The combination of these factors was predictive for patient's outcome.

Interestingly, even in this special group of patients death of disease was a rare event, with high cancerspecific survival rates of $90 \%$ and $85 \%$ after 5 and 10 years, respectively, whereas biochemical progression-free rates in the same group were as low as $65 \%$ at 5 years and $52 \%$ at 10 years.

The combination of several risk factors, expectedly, led to reduced progression-free and survival rates. In summary, radical prostatectomy is a viable option even for high-risk patients.

Dr. Andreas Bohle

Professor of Urology

HELIOS Agnes Karll Hospital

Bad Schwartau, Germany

E-mail:boehle@urologie-bad-schwartau.de

\section{NEUROLOGY \& FEMALE UROLOGY}

doi: $10.1590 / S 1677-55382010000600028$

\section{Functional results after tape removal for chronic pelvic pain following tension-free vaginal tape or transobturator tape}

Rigaud J, Pothin P, Labat JJ, Riant T, Guerineau M, Le Normand L, Glemain P, Robert R, Bouchot O

Urology Clinic, Hôtel-Dieu, Nantes University Hospital Centre, Nantes, France

J Urol. 2010; 184: 610-5

Purpose: The incidence of pelvic pain after placement of a suburethral sling for incontinence ranges between $0 \%$ and $30 \%$. The management of this chronic pain after suburethral sling placement is complex and to our knowledge no consensus has been reached. We evaluated the functional results after removal of the suburethral tape responsible for chronic pelvic pain.

Materials and Methods: From November 2004 to August 2009, 32 patients undergoing removal of suburethral tape causing chronic pelvic and perineal pain at our department were prospectively followed. Patients were divided according to the type of suburethral sling into the transobturator tape group (15 patients) and the tension-free vaginal (retropubic) tape group (17 patients). In the TVT group tape removal was performed using 
transperitoneal laparoscopy in every patient. In the TOT group tape removal was performed via a transvaginal approach possibly associated with a unilateral or bilateral incision in the proximal part of the thigh. Pain was evaluated by a visual analogue scale from 0 -no pain to 10 -maximal pain.

Results: The surgical exploration of suburethral tape responsible for chronic, treatment refractory pelvic pain revealed in most cases an abnormal tape position or excessive tape traction. In the overall population tape removal provided improvement of pain (at least 50\% improvement of the visual analogue scale score) in $68 \%$ with a mean followup of 10 months. Mean visual analogue scale score was $7.3+/-1.5$ before surgery and 3.4 $+/-3$ after surgery. However, recurrence of incontinence was observed in $22 \%$ of cases. No significant difference was demonstrated in terms of functional results according to the type of tape insertion.

Conclusions: The surgical removal of suburethral tape improved pain in $68 \%$ of patients but with a risk of recurrence of urinary incontinence in $22 \%$.

\section{Editorial Comment}

The authors review their experience in addressing chronic pelvic pain following the placement of a tension free vaginal tape or transobturator tape. Notable findings included that only approximately two-thirds of the patients were able to have a $>50 \%$ improvement in their pain control with resection of the tape. In addition, 1 in 5 patients had a return of their incontinence.

The physicians noted that the onset of pain after the placement of the tape was exceedingly rapid thus assisting the reader in linking the rapid temporal nature of this iatrogenic pain to the surgery. Of interest, is that cystoscopy, CT scan, and MRI's were all normal and thus are of potentially little assistance in evaluating this pain syndrome. For the TVT tapes, the authors utilized laparoscopy while for anatomic reasons with the transobturator tape, removals were performed transvaginally. Upon examination of the cause of pain with the TOT tape, it was noted that this was most likely to be secondary to a myofascial syndrome from muscle response to the tape passing through the body of the muscle.

Important take home messages include that the rapid appearance of pain after the placement of a suburethral tape should embolden the surgeon to promptly proceed with removal prior to the period of tissue ingrowth and that imaging studies with this temporal associated pain are of limited value. That the authors found that they had approximately $20 \%$ recurrence of incontinence after tape removal is understandable in view of similar findings of a very similar incontinence rate after simple sling incision for relief of urinary retention following pubovaginal slings (1). Strong consideration for long term physical therapy may be of value in view of the identified myofascial component and the less than $100 \%$ response to surgery for the pain.

\section{Reference}

1. Thiel DD, Pettit PD, McClellan WT, Petrou SP: Long-term urinary continence rates after simple sling incision for relief of urinary retention following fascia lata pubovaginal slings. J Urol. 2005; 174: 1878-81.

Dr. Steven P. Petrou

Professor of Urology, Associate Dean Mayo School of Graduate Medical Education

Jacksonville, Florida, USA

E-mail:petrou.steven@mayo.edu 